\title{
The Effect of Inhibitors on the Oxygen Kinetics of Terminal Oxidases of Tetrahymena pyriformis ST
}

\author{
By DAVID LLOYD, ${ }^{*}$ BODIL KRISTENSEN ${ }^{2}$ AND HANS DEGN \\ 1 Department of Microbiology, University College, Newport Road, \\ Cardiff CF2 1TA, Wales \\ ${ }^{2}$ Institute of Biochemistry, Odense University, DK-5230, Odense M, Denmark
}

(Received 3 April 1980; revised 26 May 1980)

\begin{abstract}
Respiration of early-exponential phase cultures of the ciliate protozoon Tetrahymena pyriformis is inhibited in two stages with increasing concentrations of cyanide. Up to 40 to $50 \%$ inhibition occurs at low concentrations $(<15 \mu \mathrm{M})$. Maximal inhibition was obtained with $300 \mu$ M-cyanide; at this concentration $20 \%$ of the respiration was still unaffected. Competitive inhibition of respiration by CO occurs $\left(K_{\mathrm{i}}=4.25 \mu \mathrm{M}\right)$; azide inhibition of oxygen consumption is uncompetitive $\left(K_{1}=4\right.$ to $\left.9 \mathrm{mM}\right)$. A salicylhydroxamic acid-sensitive oxidase is present which is not inhibited by cyanide, azide, $\mathrm{CO}$ or $\mathrm{H}_{2} \mathrm{~S}$. Three other pathways of terminal oxidation are present which are insensitive to azide, $\mathrm{CO}$ and salicylhydroxamic acid. One of these is cyanide- and sulphide-sensitive, a second is cyanide-insensitive but sulphide-sensitive, and a third is cyanide-sensitive but sulphide-insensitive; these pathways may not possess unique terminal oxidases. No oxidase with a very low affinity for oxygen was detected, but the overall affinity for oxygen of $T$. pyriformis in the absence of inhibitors $\left(K_{\mathrm{m}}=2.9 \mu \mathrm{M}\right)$ is lower than that of some other protozoa.
\end{abstract}

\section{INTRODUCTION}

Many organisms possess terminal oxidases other than cytochrome $a+a_{3}$, the classical oxidase of the phosphorylating respiratory chain of eukaryotes (Degn et al., 1978). Studies of the effects of respiratory inhibitors on the oxygen consumption of various protozoa have demonstrated a number of different alternative terminal oxidases; both trypanosomes and the amoeba Acanthamoeba castellanii can have at least two terminal oxidases acting alongside cytochrome $c$ oxidase (Hill, 1976; Hill \& Degn, 1977; Edwards \& Lloyd, 1978; Lloyd et al., 1979).

The respiration of the ciliate protozoon Tetrahymena pyriformis has several unusual features and has been the subject of study since Baker \& Baumberger (1941) noted its atypical $a$-type cytochromes. Subsequent investigators reported that mammalian cytochrome $c$ was not oxidized by cell-free homogenates or mitochondrial preparations, but that substrate oxidations were inhibited by azide, CO and cyanide (Ryley, 1952; Keilin \& Ryley, 1953; Eichel, 1954; Kobayashi, 1965; Van de Vijver, 1966). Functional evidence for the role of cytochrome $a_{620}$ as the main terminal oxidase of the mitochondria of $T$. pyriformis was provided by measurement of the kinetics of its reaction with oxygen (Turner et al., 1971; Lloyd \& Chance, 1972). A claim for the presence of a mitochondrial cytochrome $o$ (Perlish $\&$ Eichel, 1971) is inconclusive, and there is no evidence in this ciliate for a functional role for any $b$-type cytochrome as a terminal oxidase (Lloyd \& Chance, 1972; Kilpatrick \& Erecińska, 1977). An oxidase sensitive to inhibition by salicylhydroxamic acid has recently been demonstrated in T. pyriformis by Eichel \& Stearns (1977). 
In the present paper we describe the effects of cyanide, azide, $\mathrm{CO}, \mathrm{H}_{2} \mathrm{~S}$ and salicylhydroxamic acid on the respiration of intact organisms and suggest that at least three different terminal oxidases may function in $T$. pyriformis.

\section{METHODS}

Abbreviations. CCCP, Carbonyl cyanide $m$-chlorophenylhydrazone; SHAM, salicylhydroxamic acid.

Maintenance and growth of organisms. Tetrahymena pyriformis ST was maintained and grown axenically on $2 \%(\mathrm{w} / \mathrm{v})$ proteose peptone (Difco), $0.1 \%(\mathrm{w} / \mathrm{v})$ liver digest (Oxoid) with shaking at $30^{\circ} \mathrm{C}$ exactly as described previously (Lloyd et al., 1971). Dilution of cultures was with cell-free supernatants ('conditioned growth media'). Organisms were counted in a Sedgewick-Rafter cell (Graticules Ltd, Tonbridge, Kent) after fixation in $4 \%(\mathrm{v} / \mathrm{v})$ aqueous formaldehyde and suitable dilution in water.

Measurements of oxygen-consumption rates. Measurements of respiration were made at $30{ }^{\circ} \mathrm{C}$ by three different methods. (1) In a conventional closed reaction vessel (total vol. $2 \mathrm{ml}$ ) fitted with an oxygen electrode (Rank Brothers, Bottisham, Cambridge). (2) In an open electrode system at $30^{\circ} \mathrm{C}$ (total vol. $4 \cdot 5 \mathrm{ml}$ ) (Degn \& Wohlrab, 1971), modified as described by Petersen \& Degn (1978) to permit on-line computer control of time-dependent gradients of dissolved oxygen. The details of this method were described by Lloyd $e t$ al. (1979) and by Degn et al. (1980). (3) In an open system at $30^{\circ} \mathrm{C}$ (total vol. $4.0 \mathrm{ml}$ ) using a mass spectrometer with membrane inlet to measure steady-state concentrations of dissolved oxygen (L undsgaard et al., 1976). The system was based on a MS10 mass spectrometer fitted with a S10 sorption pump, a P25 ion pump (Kratos Ltd, A.E.I. Scientific Instruments, Manchester) and a peak detector which repeatedly scanned a narrow range around the desired mass-peak (Lundsgaard \& Petersen, 1974). The liquid sample was separated from the vacuum by a $12 \mu \mathrm{m}$ thick Teflon membrane (type D602; Radiometer, Copenhagen). The calibration procedure was as described by Lundsgaard \& Petersen (1974).

Inhibitors. Fixed concentrations of $\mathrm{CO}$ or $\mathrm{H}_{2} \mathrm{~S}$ in cell suspensions were established by mixing fixed proportions with $\mathrm{N}_{2}$ and allowing the resulting mixtures to enter the air $/ \mathrm{N}_{2}$ gas stream to the open reaction vessel (Petersen, 1977). KCN and $\mathrm{NaN}_{3}$ were used as aqueous solutions; salicylhydroxamic acid (SHAM) was dissolved in ethanol.

\section{RESULTS}

Inhibition of respiration by cyanide and azide in a closed electrode system

Successive additions of cyanide to early-exponential phase cells in growth medium ( $\mathrm{pH}$ 7.5) gave a complex inhibitory response (Fig. $1 \mathrm{a}$ ). At $13 \mu \mathrm{M}$-cyanide $50 \%$ inhibition of respiration was produced, and further inhibition was not observed until more than $30 \mu \mathrm{M}$ cyanide was added. Maximal inhibition was obtained with $300 \mu \mathrm{M}$-cyanide and the residual oxygen consumption accounted for $20 \%$ of the total respiration. Addition of $1 \mathrm{~mm}$ SHAM before the cyanide titration did not alter the biphasic nature of the response to cyanide but increased the degree of inhibition by cyanide by about $10 \%$ at all concentrations. Azide ( $25 \mathrm{mM})$ added after cyanide $(550 \mu \mathrm{M})$ gave no further inhibition. SHAM $(1 \mathrm{mM})$ added after cyanide $(550 \mu \mathrm{M})$ and azide $(25 \mathrm{mM})$ gave further inhibition $(10 \%$ of the uninhibited rate of oxygen consumption). The biphasic titration curve for cyanide inhibition was still observed when the respiration was uncoupled from energy conservation by adding $0.5 \mu \mathrm{M}$-carbonyl cyanide $m$-chlorophenylhydrazone (CCCP) but was not evident with lateexponential phase organisms (Fig. $1 a$ ).

Inhibition by azide was less than $50 \%$ even at high concentrations $(30 \mathrm{~mm})$ (Fig. $1 \mathrm{~b})$; addition of $1 \mathrm{~mm}$-cyanide at this high azide concentration gave almost $90 \%$ inhibition, and addition of 1 mM-SHAM then inhibited about half of the residual respiration. Similar results were obtained with uncoupled cell suspensions (in the presence of $0.5 \mu \mathrm{M}-\mathrm{CCCP}$ ). When azide (up to $30 \mathrm{mM}$ ) was added in the presence of $20 \mu \mathrm{M}$-cyanide, there was only a small increase of inhibition ( $<5 \%$ ) above that initially caused by cyanide alone (Fig. $1 b$ ).

Similar results were obtained for all three respiratory inhibitors with cells washed and resuspended in $67 \mathrm{~mm}$-potassium phosphate buffer $\mathrm{pH} 7 \cdot 5$. 


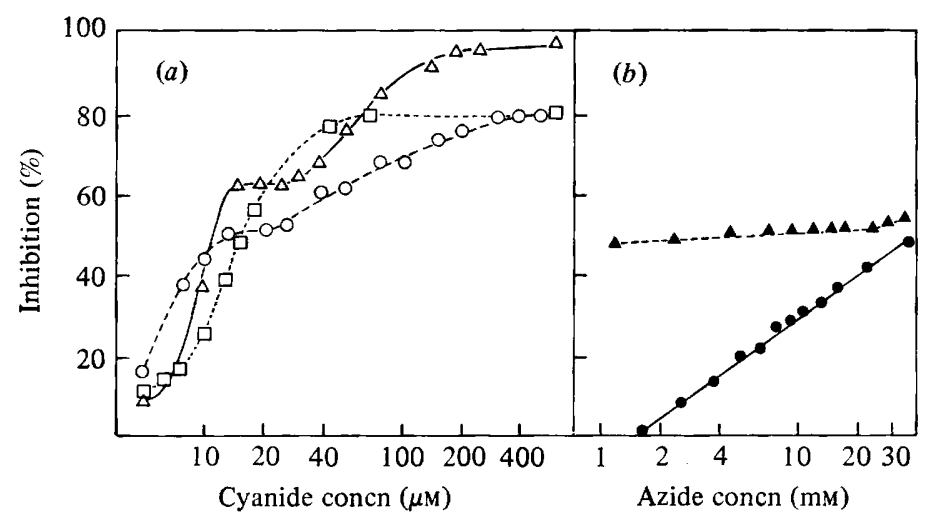

Fig. 1. Effects of cyanide and azide on the respiration of Tetrahymena pyriformis. Inhibition of respiration is expressed as a function of cyanide or azide concentration, the control ( $0 \%)$ being the respiration rate in the absence of any inhibitor. (a) Effect of cyanide on the respiration of an earlyexponential phase culture $\left(6.4 \times 10^{4}\right.$ organisms $\left.\mathrm{ml}^{-1}\right)$ in the absence $(O)$ or presence $(\Delta)$ of $0.5 \mu \mathrm{M}$ CCCP, and of a late-exponential phase culture $\left(8 \times 10^{5}\right.$ organisms $\left.\mathrm{ml}^{-1}\right)(\square)$. (b) Effect of azide in the absence ( $)$ or presence of $20 \mu \mathrm{M}$-cyanide $(\Delta)$ on the respiration of an early-exponential phase culture. Measurements were made in the closed electrode system on organisms in growth medium; the results are typical of triplicate experiments.

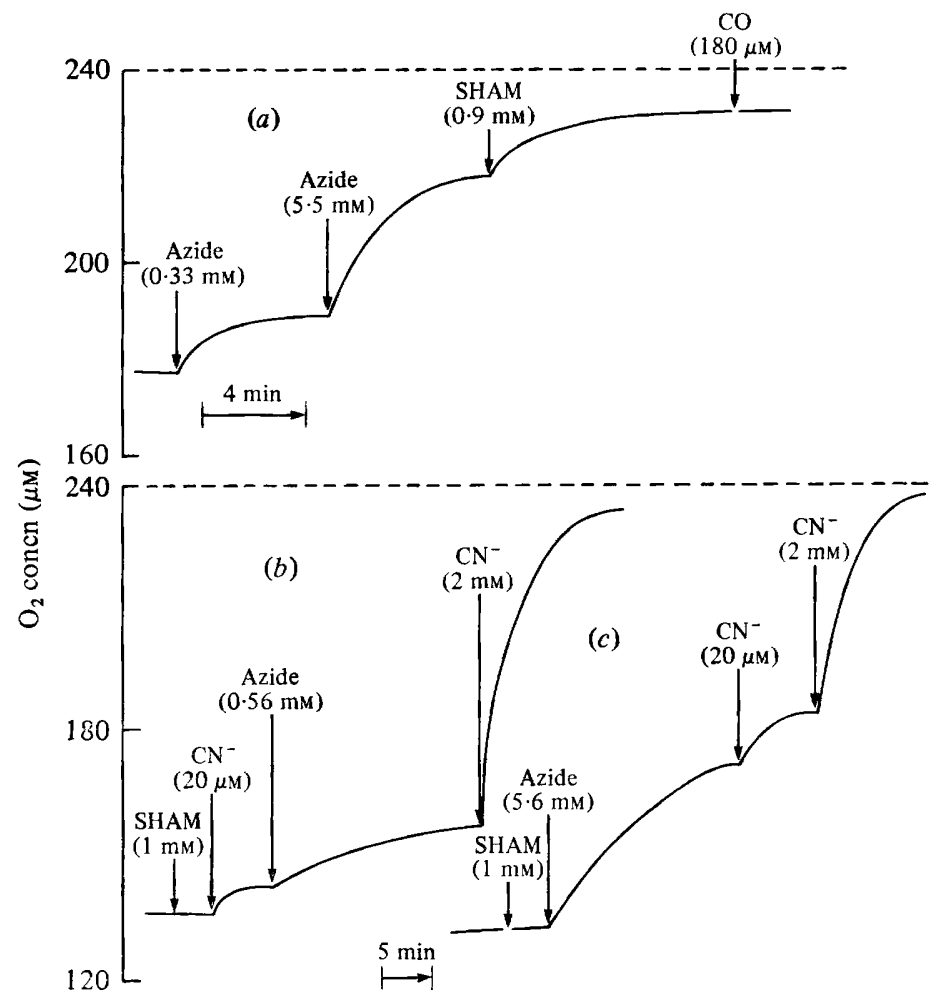

Fig. 2. Effects of respiratory inhibitors on steady-state oxygen concentrations in cultures of

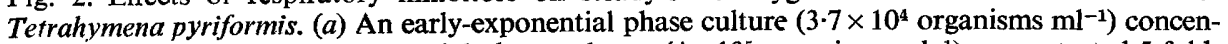
trated 20 -fold. $(b, c)$ Late-exponential phase cultures $\left(4 \times 10^{5}\right.$ organisms $\left.\mathrm{ml}^{-1}\right)$ concentrated 5 -fold. Measurements were made in the open electrode system on organisms in growth medium; the results are typical of triplicate experiments. 


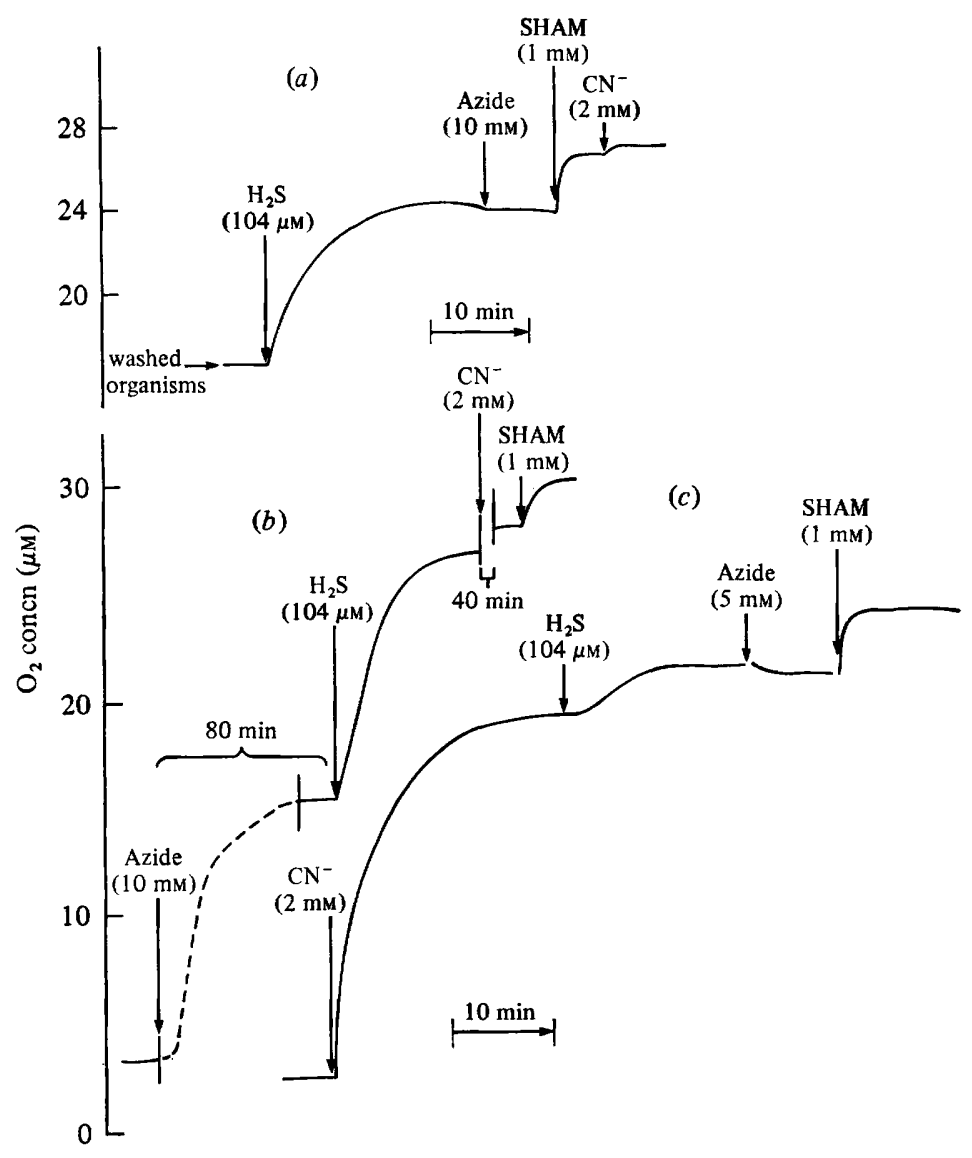

Fig. 3. Steady-state measurements of oxygen concentration in non-growing suspensions of Tetrahymena pyriformis in the open system measured by mass spectrometry. In (a) a late-exponential phase culture $\left(8 \times 10^{5}\right.$ organisms $\left.\mathrm{ml}^{-1}\right)$ was harvested and the organisms were washed once and resuspended in $67 \mathrm{mM}$-potassium phosphate buffer $\mathrm{pH} 7.5$ at a density of $10^{4}$ organisms $\mathrm{ml}^{-1}$. In $(b)$ and $(c)$, organisms were obtained in an identical way from an early-exponential phase culture $(7 \cdot 8 \times$ $10^{4}$ organisms $\mathrm{ml}^{-1}$ ). The gas phase contained $2.5 \% \mathrm{O}_{2}$ in $\mathrm{N}_{2}$ throughout the experiments; $\mathrm{H}_{2} \mathrm{~S}$ $\left(0.25 \%\right.$ in $\left.\mathrm{N}_{2}\right)$ was added as indicated into the gas stream entering the open system. Results are typical of duplicate experiments.

\section{Steady-state oxygen measurements in the presence of respiratory inhibitors}

A second method of investigating the effects of respiratory inhibitors confirmed the diversity of terminal oxidases in $T$. pyriformis. Inhibition of respiration increases the steadystate oxygen concentration in the liquid phase in the open electrode system and gives an upward deflection on the traces. Figure 2 shows that, as is the case for many organisms (Degn et al., 1978), the effect of SHAM was observable only when this inhibitor was added after cyanide or azide. Figure $2(a)$ shows that $180 \mu \mathrm{M}-\mathrm{CO}$ gave no further inhibition after $5.8 \mathrm{~mm}$-azide and 0.9 mM-SHAM had been added. Cyanide ( $2 \mathrm{mM})$ added after low (0.56 mM) concentrations of azide in the presence of 1 mM-SHAM gave extensive but not quite complete inhibition of cell respiration (Fig. $2 b$ ).

Studies of respiratory inhibition by $\mathrm{H}_{2} \mathrm{~S}$ require the use of a mass spectrometer as this compound interferes with electrode-based oxygen assays; reaction of $\mathrm{H}_{2} \mathrm{~S}$ with constituents of the growth medium necessitated the use of washed cell suspensions. Inhibition of the endogenous respiration of organisms (suspended in $67 \mathrm{~mm}$-potassium phosphate buffer $\mathrm{pH}$ 


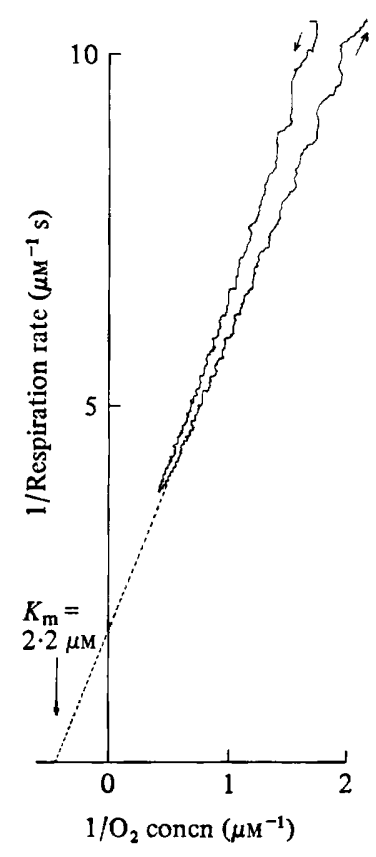

Fig. 4

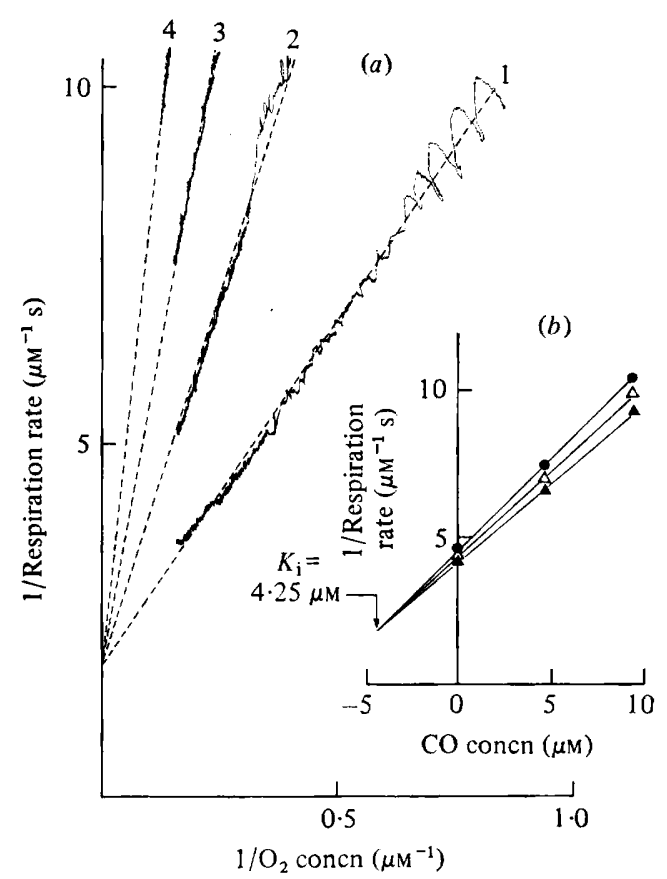

Fig. 5

Fig. 4. Reciprocal plots of respiration rate against oxygen concentration for an early-exponential phase culture of Tetrahymena pyriformis. The culture was harvested at a density of $7 \cdot 7 \times 10^{4}$ organisms $\mathrm{ml}^{-1}$ and resuspended at ten times this concentration in conditioned growth medium. Arrows indicate increasing (top trace) and decreasing (lower trace) time gradients of dissolved oxygen. Similar results were obtained in 15 experiments.

Fig. 5. (a) Reciprocal plots of respiration rate against decreasing oxygen concentration in the presence of various fixed $\mathrm{CO}$ concentrations for an early-exponential phase culture of Tetrahymena pyriformis: curve 1 , uninhibited culture; $2,4 \cdot 7 \mu \mathrm{M}-\mathrm{CO} ; 3,9 \cdot 4 \mu \mathrm{M}-\mathrm{CO} ; 4,14 \cdot 1 \mu \mathrm{M}-\mathrm{CO}$. The culture was harvested at a density of $6.0 \times 10^{4}$ organisms $\mathrm{ml}^{-1}$ and resuspended at five times this concentration in conditioned growth medium. Similar results were obtained in three experiments.

(b) Plot of the reciprocal rate of oxygen uptake against $\mathrm{CO}$ concentration at various fixed oxygen concentrations. Data from $(a): 0,4 \cdot 0 \mu \mathrm{M}-\mathrm{O}_{2} ; \triangle, 4 \cdot 6 \mu \mathrm{M}-\mathrm{O}_{2} ; \Delta, 5 \cdot 33 \mu \mathrm{M}-\mathrm{O}_{2}$.

7.5) by $104 \mu \mathrm{M}-\mathrm{H}_{2} \mathrm{~S}$ completely removed the azide-sensitive portion of respiration. Subsequent addition of 1 mM-SHAM gave further inhibition, as did 2 mM-cyanide (Fig. $3 a$ ). Changing the order of additions confirmed that $\mathrm{H}_{2} \mathrm{~S}$ gave more complete inhibition than $10 \mathrm{~mm}$-azide and that the respiration which persisted in the presence of these two inhibitors had separate cyanide-sensitive and SHAM-sensitive components (Fig. 3b). A sulphidesensitive, cyanide-insensitive component of respiration was also detected (Fig. $3 c$ ).

\section{Steady-state oxygen kinetics of terminal oxidases}

Time gradients over a concentration range 0.5 to $3 \mu \mathrm{M}$-oxygen were used to investigate the dependence of the respiration rate of organisms on oxygen tension in the growth medium. In the absence of inhibitors the Lineweaver-Burk plots of reciprocal respiration rate against reciprocal oxygen concentration were linear when the oxygen tension was decreased with time (Fig. 4). Plots produced by increasing oxygen tension from anaerobiosis were always convex downwards. Values for the apparent $K_{\mathrm{m}}$ for oxygen were therefore obtained by extrapolation of plots from experiments in which the steady-state level of dissolved oxygen was decreased with time. The apparent $K_{\mathrm{m}}$ for oxygen was $2.9 \pm 0.9 \mu \mathrm{M}$ (mean \pm S.D., 10 

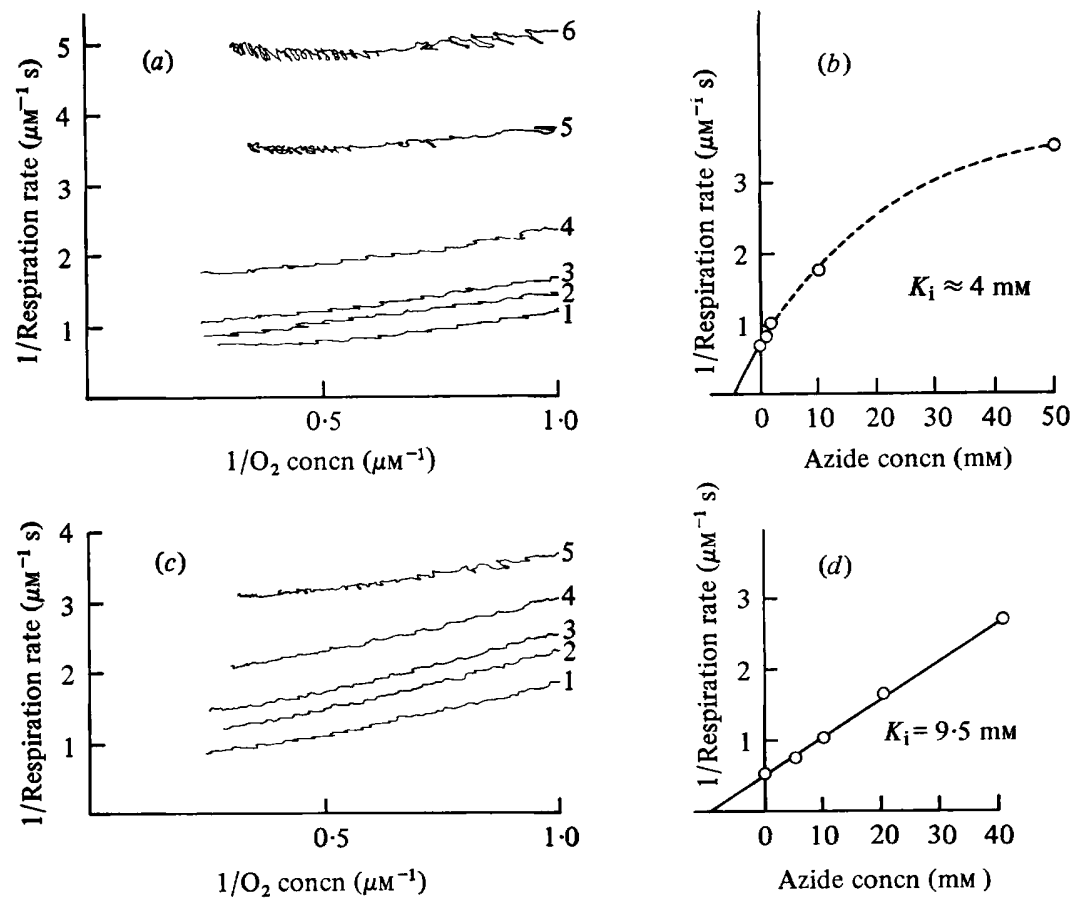

Fig. 6. (a,c) Reciprocal plots of respiration rate against decreasing oxygen concentration in the presence of various fixed azide concentrations for early-exponential phase $(a)$ and late-exponential phase (c) cultures of Tetrahymena pyriformis. (a) Curve 1, uninhibited culture; 2, 0.5 mm-azide; 3, 2 mM-azide; 4, 10 mm-azide; 5, 50 mm-azide; 6, 50 mM-azide plus 1 mM-SHAM. The culture was harvested at a density of $7 \cdot 8 \times 10^{4}$ organisms $\mathrm{ml}^{-1}$ and resuspended at seven times this concentration in conditioned growth medium. Similar results were obtained in three experiments. (c) Curve 1, uninhibited culture; 2, 5 mM-azide; 3, 10 mM-azide; 4, 20 mM-azide; 5, 40 mm-azide. The culture was harvested at a density of $8 \times 10^{5}$ organisms $\mathrm{ml}^{-1}$ and resuspended at three times this concentration in conditioned growth medium.

$(b, d)$ Plots of the corresponding reciprocals of maximal respiration rates at various azide concentrations. Data are taken from $(a)$ and $(c)$, respectively.

determinations) and was not significantly different for organisms at the early- or lateexponential phases of growth ( $<10^{5}$ or $>3 \times 10^{5}$ organisms $\mathrm{ml}^{-1}$, respectively).

The non-linear Lineweaver-Burk plots obtained when the oxygen tension was increased with time may result from a control mechanism which causes a retardation in the response of the respiratory system to an increased oxygen concentration. Thus each small stepwise increase of the oxygen tension in the gas phase leads to an overshoot of the oxygen concentration in the culture before the attainment of the new steady-state. A similar phenomenon has been observed in other protozoa and with yeasts ( $\mathrm{H}$. Degn, unpublished observations).

Figure 5(a) shows that the inhibition of respiration by various fixed partial pressures of $\mathrm{CO}$ at low oxygen concentrations was strictly competitive. Secondary plots gave a $K_{\mathrm{i}}$ value of $4 \cdot 25 \mu \mathrm{M}$ for inhibition by $\mathrm{CO}$ (Fig. $5 b$ ). Similar results were obtained with early- or lateexponential phase organisms.

In the presence of various fixed concentrations of azide (up to $10 \mathrm{~mm}$ ) a series of parallel Lineweaver-Burk plots were obtained with organisms from both early- andilate-exponential phase cultures (Fig. $6 a, c$ ). At the highest azide concentrations (40 to $50 \mathrm{~mm}$ ) departures from linearity were observed. Secondary plots of intercepts against inhibitor concentration gave a non-linear relationship for early-exponential phase organisms with a $K_{1}$ value of about $4 \mathrm{mM}$ (Fig. $6 b$ ) and a linear dependence for late-exponential phase organisms $\left(K_{1}=9 \cdot 5\right.$ 


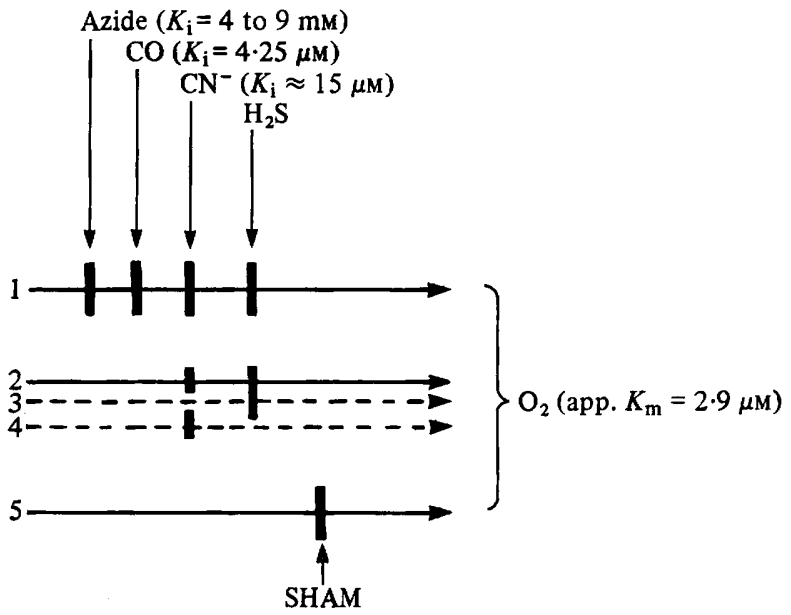

Fig. 7. Inhibitor-sensitivities of pathways of terminal oxidation in Tetrahymena pyriformis.

mM; Fig. $6 d$ ). Addition of 1 mM-SHAM to early-exponential phase cultures containing 50 mM-azide (Fig. $6 a$ ) gave further inhibition of respiration over the range of oxygen concentrations studied (1 to $4 \mu \mathrm{M}$ ).

\section{DISCUSSION}

Studies of the effects of respiratory inhibitors on the respiration of intact $T$. pyriformis confirm that the process of terminal oxidation in this protozoon can be resolved into five distinct pathways (terminating in at least three oxidases); the simplest hypothetical representation of these (neglecting the possibilities for confluence and branching) is shown in Fig. 7. Pathway 1 is inhibited by azide $\left(K_{\mathrm{i}}=4\right.$ to $\left.9 \mathrm{mM}\right), \operatorname{CO}\left(K_{\mathrm{i}}=4 \cdot 25 \mu \mathrm{M}\right)$, cyanide $\left(K_{\mathrm{i}} \approx\right.$ $15 \mu \mathrm{M})$ and $\mathrm{H}_{2} \mathrm{~S}$. A second major pathway is inhibited by much higher cyanide concentrations and by $\mathrm{H}_{2} \mathrm{~S}$. Pathway 3 is $\mathrm{H}_{2} \mathrm{~S}$-sensitive but cyanide-insensitive, while a fourth pathway is inhibited only by high concentrations of cyanide ( 1 to $2 \mathrm{~mm}$ ). Pathway 5 is unaffected by any of these inhibitors but is sensitive to inhibition by SHAM. Under the growth conditions employed pathways 3,4 and 5 account for only a small proportion of the total electron flux, and further evidence is required (e.g. from other specific inhibitors) that pathways 3 and 4 possess unique terminal oxidases.

Linear Lineweaver-Burk plots of reciprocal respiration rate against reciprocal oxygen concentration are not to be expected for a mixture of oxidases with different kinetic properties. That linear plots are nevertheless obtained suggests either that these properties are very similar for the individual oxidases, or that one pathway of electron transport is dominant under the conditions of measurement.

No indication of the presence of an oxidase with a low affinity for oxygen was observed; the azide-insensitive (but cyanide-sensitive) component observed here is thus different from the low-affinity oxidase of Acanthamoeba castellanii (Lloyd et al., 1979). The overall affinity of the respiratory system of $T$. pyriformis for oxygen is, however, lower than those measured for several other eukaryotes. The value of $2.9 \mu \mathrm{M}$-oxygen for the apparent $K_{\mathrm{m}}$ of this ciliated protozoon compares with $<1 \mu \mathrm{M}$ for $A$. castellanii (Lloyd et al., 1979), $0 \cdot 1 \mu \mathrm{M}$ for the tsetse fly vector form of a trypanosome (Hill, 1978) and $<1.5 \mu \mathrm{M}$ for baker's yeast $(\mathrm{H}$. Degn, unpublished results).

Further work is necessary to decide whether each of the five pathways of terminal respiration, identified by successive inhibitor additions, represent five different terminal 
oxidases. It has been shown that cytochrome $a_{620}$ is kinetically competent as a terminal oxidase (Turner et al., 1971), and spectral data indicate that this cytochrome reacts with CO and cyanide (Lloyd \& Chance, 1972) and with azide (D. Lloyd, unpublished results). The present work suggests that this oxidase (pathway 1) is also inhibited uncompetitively by azide and by $\mathrm{H}_{2} \mathrm{~S}$. The high concentrations of azide required for inhibition may reflect the presence of a permeability barrier to this inhibitor in whole organisms: inhibition in the presence of CCCP shows that azide is not acting as an inhibitor of ATP synthetase. Component 5 has previously been identified in $T$. pyriformis as a SHAM-sensitive oxidase (Eichel \& Stearns, 1977), highly active in organisms grown in media supplemented with metal ions (Stearns et al., 1978). Components 2, 3 and 4 may represent novel alternative terminal oxidases or modified forms of component 1 , possibly in specialized membrane microenvironments. Further investigations are necessary to identify the alternative pathways of electron transport in $T$. pyriformis and the changes in apportionment of electron flux between these during growth in batch cultures.

D.L. thanks members of the Institute of Biochemistry at Odense for their help and hospitality and Miss J. Ball for technical help.

\section{REFERENCES}

Baker, E. G. S. \& Baumberger, J. P. (1941). The respiratory rate and the cytochrome content of a ciliated protozoan (Tetrahymena geleii). Journal of Comparative and Cellular Physiology 17, 285303.

Degn, H. \& Wohlrab, H. (1971). Measurement of steady state values of respiration rate and oxidation levels of respiratory pigments at low oxygen tensions. A new technique. Biochimica et biophysica acta 245, 347-355.

Degn, H., Lloyd, D. \& Hill, G. C. (1978). Functions of Alternative Terminal Oxidases, pp. 196. Oxford: Pergamon.

Degn, H., Lundsgaard, J. S., Petersen, L. C. \& Ormicki, A. (1980). Polarographic measurement of steady state kinetics of oxygen uptake by biochemical samples. Methods of Biochemical Analysis 26, 47-77.

Edwards, S. W. \& Lloyd, D. (1978). Properties of mitochondria isolated from cyanide-sensitive and cyanide-stimulated cultures of Acanthamoeba castellanii. Biochemical Journal 174, 203-211.

EICHEL, H. J. (1954). Studies on the oxidation of succinic acid by cell-free homogenates of Tetrahymena pyriformis $\mathrm{S}$ and W. Journal of Biological Chemistry 206, 159-169.

Eichel, H. J. \& Stearns, F. M. (1977). Cyanideinsensitive mitochondrial respiratory pathway in intact Tetrahymena and its specific inhibitor hydroxamic acid. Federation Proceedings 36, 904.

HILL, G. C. (1976). Electron transport systems in Kinetoplastida. Biochimica et biophysica acta 456, 149-193.

Hill, G. C. (1978). Characterization of the electron transport systems present during differentiation of African trypanosomes. In Functions of Alternative Terminal Oxidases, pp. 67-77. Edited by $\mathrm{H}$. Degn, D. Lloyd \& G. C. Hill. Oxford: Pergamon.

Hill, G. C. \& DEGN, H. (1977). Steady-state oxygen kinetics of terminal oxidases in Trypanosoma mega. Journal of Protozoology 24, 563-565.
Keilin, D. \& Ryley, J. F. (1953). Haemoglobin in protozoa. Nature, London 172, 451.

Kilpatrick, L. \& ERecińska, M. (1977). Mitochondrial respiratory chain of Tetrahymena pyriformis. The thermodynamic and spectral properties. Biochimica et biophysica acta 460 , 346-363.

KoBAYASHI, S. (1965). Preparation and properties of mitochondria from the ciliated protozoon Tetrahymena. Journal of Biochemistry 58, 444-457.

LloYd, D. \& Chance, B. (1972). The cytochromes of mitochondria from Tetrahymena pyriformis strain ST. Biochemical Journal 128, 1171-1182.

Lloyd, D., Brightwell, R., Venables, S. E., ROACH, G. I. \& TuRNer, G. (1971). Subcellular fractionation of Tetrahymena pyriformis ST by zonal centrifugation: changes in activities and distribution of enzymes during the growth cycle and on starvation. Journal of General Microbiology 65, 209-223.

Lloyd, D., Edwards, S. W., Kristensen, B. \& DeGN, H. (1979). The effect of inhibitors on the oxygen kinetics of terminal oxidases of Acanthamoeba castellanii. Biochemical Journal 182, 1115.

Lundsgaard, J. S. \& Petersen, H. A. (1974). Measurement of oxygen by mass spectrometry. Journal of Physics E-Scientific Instruments 7, 524.

Lundsgaard, J. S., Petersen, L. C. \& Degn, H. (1976). Mass spectrometric determination of oxygen kinetics in biochemical systems. In Measurement of Oxygen, pp. 168-183. Edited by H. Degn, I. Balslev \& R. Brook. Amsterdam: Elsevier.

Perlish, J. S. \& Eichel, H. J. (1971). A succinateand DPNH-reducible $o$-type cytochrome in mitochondrial preparations from Tetrahymena pyriformis. Biochemical and Biophysical Research Communications 44, 973-980. 
Petersen, L. C. (1977) The effect of inhibitors on the oxygen kinetics of cytochrome $c$ oxidase. Biochimica et biophysica acta 460, 299-307.

Petersen, L. C. \& Degn, H. (1978). Steady-state kinetics of laccase from Rhus vernicifera. Biochimica et biophysica acta 526, 85-92.

RYLEY, J. F. (1952). Studies on the metabolism of the protozoa. III. Metabolism of the ciliate Tetrahymena pyriformis (Glaucoma pyriformis). Biochemical Journal 52, 483-492.

Stearns, F. M., DeMaio, R. A. \& Eichel, H. J.
(1978). Occurrence of cyanide-resistant respiration in Tetrahymena cells grown with various metals. Federation Proceedings 37, 1516.

Turner, G., Lloyd, D. \& Chance, B. (1971). Electron transport in phosphorylating mitochondria from Tetrahymena pyriformis strain ST. Journal of General Microbiology 65, 359-374.

VAN DE VIJVER, G. (1966). Studies on the metabolism of Tetrahymena pyriformis GL. II. Some properties of the terminal oxidation. Enzymologia 31, 382-391. 